\begin{tabular}{|c|}
\hline $\begin{array}{r}\text { PRAMANA } \\
\text { - journal of } \\
\text { physics }\end{array}$ \\
\hline
\end{tabular}

\title{
Anomalous top magnetic couplings
}

\author{
G GONZÁLEZ-SPRINBERG ${ }^{1}$, R MARTINEZ ${ }^{2, *}$ and JORGE VIDAL ${ }^{3}$ \\ ${ }^{1}$ Instituto de Física, Facultad de Ciencias, Universidad de la República, Montevideo, Uruguay \\ ${ }^{2}$ Departamento de Física, Universidad Nacional, Bogotá, Colombia \\ ${ }^{3}$ Departament de Física Teòrica Universitat de València, Burjassot,València, Spain \\ *Corresponding author. E-mail: remartinezm@unal.edu.co
}

Abstract. The real and imaginary parts of the one-loop electroweak contributions to the left and right tensorial anomalous couplings of the $t b W$ vertex in the Standard Model (SM) are computed.

Keywords. Top; anomalous.

PACS Nos 14.65.Ha; 12.15.Lk

Top quark physics at the Large Hadron Collider (LHC) is an important scenario for testing physics above the electroweak scale [1,2]. Some effects related to the top anomalous couplings, both in the $t \rightarrow b W^{+}$polarized branching fractions and in single top production at the Tevatron and at the LHC, have already been studied in recent years. One-loop QCD and electroweak contributions to the $t b W$ vertex have been studied in the frame of the Standard Model (SM) [3]. The explicit dependence of the polarized branching fractions on the anomalous couplings have been computed in refs $[4,5]$.

We compute the electroweak SM contribution to the left and right 'magnetic' tensorial couplings of the $t b W$ vertex. We found that the electroweak contribution is also at the level of $10 \%$ with respect to the leading gluon exchange. For on-shell particles, the amplitude $\mathcal{M}_{t b W}$ can be written in the following way:

$$
\mathcal{M}_{t b W^{+}}=-\frac{e}{\sin \theta_{\mathrm{W}} \sqrt{2}} \epsilon^{\mu *} \bar{u}_{\mathrm{b}}\left[\frac{\mathrm{i} \sigma_{\mu \nu} q^{\nu}}{m_{\mathrm{W}}}\left(g_{\mathrm{L}} P_{\mathrm{L}}+g_{\mathrm{R}} P_{\mathrm{R}}\right)\right] u_{\mathrm{t}} .
$$

One-loop QCD gluon exchange contribution to $g_{\mathrm{R}}$ was computed in ref. [6], $g_{\mathrm{R}}^{\mathrm{QCD}}=$ $-6.61 \times 10^{-3}$. The sensitivity to $g_{\mathrm{R}}$ will be accessible to the LHC experiments $[2,5]$. The left tensorial term couples a right $b$-quark and thus it is proportional to $m_{\mathrm{b}}$. Then, constraints on $g_{\mathrm{L}}$ are stronger than $g_{\mathrm{R}}$ due to the chiral $m_{\mathrm{t}} / m_{\mathrm{b}}$ factor.

Indirect limits on $g_{\mathrm{L}}$ and $g_{\mathrm{R}}$ can be obtained from $b \rightarrow s \gamma$ [7]. The results from the analysis given in refs [8] and [9] are given in the first line of table 1; the second and third lines show $g_{\mathrm{L}}$ and $g_{\mathrm{R}}$ limits predicted for the future LHC data [5]. The LHC will improve the sensitivity to $g_{\mathrm{R}}$ by an order of magnitude compared to bounds from $b \rightarrow s \gamma$. In the same way as it is done in Tau physics [10], new asymmetry observables derived from 
Table 1. Bounds on $g_{\mathrm{R}}$ and $g_{\mathrm{L}}$.

\begin{tabular}{lccc}
\hline Reference & & $g_{\mathrm{R}}$ Bound & $g_{\mathrm{L}}$ Bound \\
\hline bs $\gamma$ & $95 \% \mathrm{CL}$ & $-0.15<g_{\mathrm{R}}<0.57$ & $-0.0015<g_{\mathrm{L}}<0.0004$ \\
Future LHC data & $2 \sigma$ & $-0.026 \leq g_{\mathrm{R}} \leq 0.031$ & $-0.058 \leq g_{\mathrm{L}} \leq 0.026$ \\
Future LHC data & $1 \sigma$ & $-0.012 \leq g_{\mathrm{R}} \leq 0.024$ & $-0.16 \leq g_{\mathrm{L}} \leq 0.16$ \\
\hline & & $g_{\mathrm{R}} \operatorname{Discovery~limit}$ & $g_{\mathrm{L}}$ Discovery limit \\
\hline Helicity fractions of the $W$ & $3 \sigma$ & $\left|\operatorname{Re}\left(g_{\mathrm{R}}\right)\right| \geq 0.056$ & $\operatorname{Re}\left(g_{\mathrm{L}}\right) \geq 0.051$ or \\
& & & $\operatorname{Re}\left(g_{\mathrm{L}}\right) \leq-0.083$ \\
bs $\gamma$ & $3 \sigma$ & $\left|\operatorname{Im}\left(g_{\mathrm{R}}\right)\right| \geq 0.115$ & $\left|\operatorname{Im}\left(g_{\mathrm{L}}\right)\right| \geq 0.065$ \\
& & $\operatorname{Re}\left(g_{\mathrm{R}}\right) \geq 0.76$ or & $\operatorname{Re}\left(g_{\mathrm{L}}\right) \geq 0.0009$ or \\
& & $\operatorname{Re}\left(g_{\mathrm{R}}\right) \leq-0.33$ & $\operatorname{Re}\left(g_{\mathrm{L}}\right) \leq-0.0019$ \\
& & & $\left|\operatorname{Im}\left(g_{\mathrm{L}}\right)\right| \geq 0.006$ \\
\hline
\end{tabular}

helicity fractions for polarized $W$ were defined for polarized top decays; the exclusion intervals derived from these observables are shown in the fourth line of table 1 . As a reference for the comparison with the LHC, they also derived as $3 \sigma$ discovery limits from $b \rightarrow s \gamma$ in ref. [9]; this is shown in the last line of table 1 .

At one loop in the SM, there is only one topology for the diagrams that contribute to the anomalous $g_{\mathrm{R}}$ and $g_{\mathrm{L}}$ : this is shown in figure $1 \mathrm{a}$. For $g_{\mathrm{R}}$ there are two diagrams that have a leading $m_{\mathrm{t}}$-mass. They are the ones in figure $1 \mathrm{~b}$ with $t h W$ and $t w_{0} W$ circulating in the loop, where $h$ is the Higgs boson and $w_{0}$ is the unphysical Z-boson. These two diagrams have top mass insertions that give a mass dependence which is of the order $1 / r_{\mathrm{W}}^{2}=1 /\left(m_{\mathrm{W}} / m_{\mathrm{t}}\right)^{2}$ with respect to the other diagrams. Some diagrams, like $b W Z$ for example, contribute to the imaginary part of $g_{\mathrm{R}}$.

The result for each contribution of the diagrams to $g_{\mathrm{R}}$ and $g_{\mathrm{L}}$ is given in table 2, with $m_{\mathrm{h}}=150 \mathrm{GeV}$. The final result for the one-loop electroweak correction is

$$
g_{\mathrm{R}}^{\mathrm{EW}}=-(0.56+1.23 i) \times 10^{-3}, \quad g_{\mathrm{L}}^{\mathrm{EW}}=-(0.92+0.14 i) \times 10^{-4} .
$$

(a)

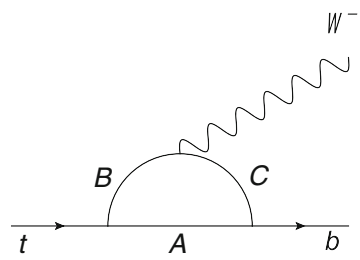

(b)

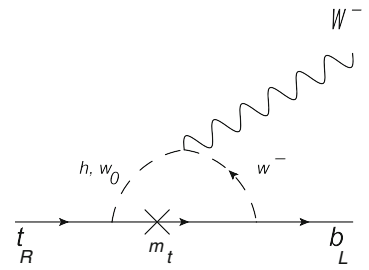

Figure 1. (a) Topology of the one-loop SM Feynman diagrams for the quantum correction to the $t \rightarrow b W^{+}$decay. (b) Leading order diagrams for $g_{\mathrm{R}}$ in the large $m_{\mathrm{t}}$ limit. 
Table 2. Electroweak contributions to $g_{\mathrm{R}}$ and $g_{\mathrm{L}}$.

\begin{tabular}{lcc}
\hline Diagram & $g_{\mathrm{R}} \times 10^{3}$ & $g_{\mathrm{L}} \times 10^{3}$ \\
\hline$t Z W$ & -1.176 & -0.0141 \\
$t h W$ & 0.220 & 0 \\
$t w^{0} w^{-}$ & 0.344 & 0.0051 \\
$h w^{-}$ & 0.462 & -0.0088 \\
$t Z w^{-}$ & -0.050 & -0.0012 \\
$t \gamma W+t \gamma w^{-}$ & 0.572 & -0.0094 \\
$b W Z$ & $-0.623-0.664 i$ & $-0.0201-0.0214 i$ \\
$b W h$ & 0 & $0.0086-0.0120 i$ \\
$b w^{+} w^{0}$ & $(1.5+11.0 i) \times 10^{-4}$ & $-0.0029-0.0167 i$ \\
$b w^{+} h$ & $-4.3+8.6 i) \times 10^{-4}$ & $-0.0019+0.0111 i$ \\
$b w^{+} Z$ & $-0.088-0.062 i$ & $-0.00039-0.00028 i$ \\
$b W \gamma+b w^{+} \gamma$ & $0.114-0.509 i$ & $-0.0270+0.0250 i$ \\
$Z t b$ & -0.397 & -0.0067 \\
$\gamma t b$ & 0.068 & 0.0115 \\
$w^{0} t b$ & $-6.8 \times 10^{-4}$ & -0.0109 \\
$h t b$ & $-6.2 \times 10^{-4}$ & -0.0135 \\
$\Sigma(E W)$ & $-0.56-1.23 i$ & $-(0.092+0.014 i)$ \\
$g t b$ & -6.61 & -1.12 \\
\hline
\end{tabular}

We note that for $g_{\mathrm{L}}^{\mathrm{EW}}$ is $8 \%$ of $g_{\mathrm{L}}^{g}$, and also that the CP violation has its origin in the electroweak diagrams. These values are to be compared with the gluon contribution that is the dominant one:

$$
g_{\mathrm{R}}^{\mathrm{g}}=-6.61 \times 10^{-3}, \quad g_{\mathrm{L}}^{\mathrm{g}}=-1.12 \times 10^{-3} .
$$

The final result for the one-loop computation in the SM is the sum of eqs (2) and (3):

$$
g_{\mathrm{R}}^{\mathrm{SM}}=-(7.17+1.23 i) \times 10^{-3}, \quad g_{\mathrm{L}}^{\mathrm{SM}}=-(1.21+0.01 i) \times 10^{-3} .
$$

The real part for the one-loop electroweak quantum correction for $g_{\mathrm{R}}$ is $8 \%$ of the leading gluon-exchange contribution. Note that the imaginary part is $17 \%$ of the one-loop $\operatorname{Re}\left(g_{\mathrm{R}}^{\mathrm{SM}}\right)$.

\section{Acknowledgements}

This work was supported by ANII-FCE-2986-Uruguay, COLCIENCIAS-Colombia, the Spanish Ministry of Science and Innovation, under grants FPA2008-03373, FPA200802878, and by Generalitat Valenciana under grant PROMETEO 2009/128.

\section{References}

[1] M Beneke et al, hep-ph/0003033

[2] W Bernreuther, J. Phys. GG35, 083001 (2008)

E Boos, L Dudko and T Ohl, Eur. Phys. J. C11, 473 (1999)

J A Aguilar-Saavedra, Nucl. Phys. B804, 160 (2008) 
[3] H S Do et al, Phys. Rev. D67, 091501 (2003)

[4] C R Chen, F Larios and C P Yuan, Phys. Lett. B631, 126 (2005)

Gabriel A Gonzalez-Sprinberg, Roberto Martinez and Jorge Vidal, J. High Energy Phys. 1107, 094 (2011)

[5] F del Águila and J A Aguilar-Saavedra, Phys. Rev. D67, 014009 (2003)

J A Aguilar-Saavedra et al, Eur. Phys. J. C804, 160 (2008)

J A Aguilar-Saavedra et al, Eur. Phys. J. C53, 689 (2008)

J Bernabéu and J A Aguilar-Saavedra, Nucl. Phys. B840, 349 (2010)

[6] C S Li, R J Oakes and T C Yuan, Phys. Rev. D43, 3759 (1991)

[7] M Jezabek and J H Kuhn, Phys. Rev. D48, 1910 (1993)

A Czarnecki, Phys. Lett. B252, 467 (1990)

C S Li, R J Oakes and T C Yuan, Phys. Rev. D43, 3759 (1991)

[8] J Alwall et al, Eur. Phys. J. C49, 791 (2007)

[9] B Grzadkowski and M Misiak, Phys. Rev. D78, 077501 (2008)

[10] J Bernabéu, G A González-Sprinberg and J Vidal, Phys. Lett. B326, 168 (1994)

J Bernabéu et al, Nucl. Phys. B436, 474 (1995) 